\title{
CCD DATA TAKING MODES AND FLATFIELDING PROBLEMS
}

\author{
S. Djorgovski \\ California Institute of Technology \\ Pasadena, CA 91125, USA. \\ M. Dickinson \\ Astronomy Department, University of California \\ Berkeley, CA 94720, USA.
}

\begin{abstract}
We briefly review some problems in the flatfielding of CCD images, in the context of three data-taking modes: stare, short scan, and drift scan. The principal sources of flatfielding imperfections are: (1) mismatch in the spectra of the astronomical sources of interest and the flatfield illumination; (2) a variety of low-level additive errors; and (3) nonlinearities of the CCD response. Residual flatfielding errors are probably the limiting factor in high-precision astronomical photometry. Flatfielding accuracies of the order of $1-2 \%$ per pixel are commonly achieved; with some effort, accuracies of $\sim 0.1 \%$ can be reached; higher accuracies require a substantial effort, or improvements in the quality of CCD chips. In general, scanning data taking modes outperform the "standard" stare mode.
\end{abstract}

The CCD revolution in astronomy is comparable in its impact to the introduction of astronomical photography a century ago. There is hardly a field of astronomy where the new optical and near-optical imaging and spectroscopy with CCD's has not brought vast qualitative and quantitative advances. Most of the success is due to the high quantum efficiency (QE) and benign noise properties of these devices, as well as to the relative ease of obtaining linear, calibrated data in a digital form with them. However, this ease is somewhat deceptive; few observers bother to extract astronomical data from their raw CCD images with the accuracy of which the devices are capable. Worse yet, inadequate preprocessing procedures can degrade the quality of the data, or introduce systematic errors which are not reflected in nominal Poissonian error-bars. For many applications, high photometric accuracy is not required, or even pursued, but it is achievable with a modicum of effort.

For the general lore about astronomical CCD's, their charms and problems, the reader is referred to the reviews by Mackay (1986) and Djorgovski (1984), the proceedings edited by Baluteau and D'Odorico (1986), the thesis by Wright (1982), many S.P.I.E. proceedings on astronomical instrumentation (e.g., volumes $264,290,445,501$, and 627), the issues $8-10$ of the vol. 26 of Optical Engineering (1987), and references therein. Many application papers often give useful advice on processing procedures. For example, the paper by Stetson and Harris (1988) is 
a model of a crowded-field stellar photometry study. The paper by Tyson (1988; and references therein) addresses faint galaxy counts at the limits of present-day technology. A truly interested reader should also try to get on the preprint mailing list of James Janesick from the JPL.

Different sources of errors will dominate in different applications (e.g, faint galaxy counts vs. standard star exposures). For example, in short exposures, readout noise and charge transfer inefficiencies will define the noise floor, whereas flatfielding errors may dominate in the high-signal regime. One should thus optimize one's data-taking and processing techniques for the particular problem at hand. There is no universal best solution for all CCD observations, although some hygienic practices are always commendable. In this paper, we deal with the CCD flatfielding and its limitations in general.

We define flatfielding as the removal of sensitivity variations of a CCD detector on all spatial scales. Different flatfield images can be used to remove the sensitivity or illumination variations at different scales: pixel-to-pixel, global gradients and vigneting, or the scales in-between. The intra-pixel variations (if important) must be modeled. Such variations, generally due to the CCD architecture and illumination patterns, are seen in the GEC CCD's (Wright 1982), but mercifully not in the popular "HST/Galileo" TI $800^{2}$ devices (Gunn, priv. comm.) In general, when observing, it is a worthwhile goal to expose one's object(s) not just on a single area of the CCD, but multiply, on various different pixels or portions of the CCD, so that residual variations partly average out or cancel. This may be achieved in a number of ways discussed below.

There are three principal modes of CCD data taking: (1) Stare or Steady, in which the telescope and the detector are in a stable pointing, tracking the object of interest. This is the standard mode, and the easiest to implement, but it gives the worst results. An expensive (in terms of observing and data reduction overhead), but highly beneficial variation on it is a mode of Multiple Exposures with Shifting and Addition; if the device is good, and enough independent exposures are taken, the flatfielding may be unnecessary (Tyson 1988). (2) Drift Scan, in which either the telescope or the CCD moves, and the charge is continuously read out, row by row, at a synchronized rate so that the charge image and the optical image always coincide (cf. Wright 1982, or Hall and Mackay 1984). Not only is the flatfielding reduced to a 1-dimensional problem (column-to-column response) by averaging the object signal over all rows of the device, but the method also removes the pesky interference fringes that occur in many systems. The bad side of this technique, from a photometric point of view, is that it is sensitive to seeing, sky brightness, and transparency variations; also, it is efficient only in long scan strips, due to the overhead "ramp-up" period during which not all pixels read out have been exposed to the sky for the same period of time. (3) Short Scan, invented by Roger Lynds (KPNO), is like (2), but the CCD is scanned over only part of its extent, typically $30-50$ rows. The ramp-up is lost, but the ramp-down is kept, and is at least partly usable. This method has practically all the advantages of drift scanning with none of its drawbacks. If the telescope is capable of precision offset guiding, or a moving-stage CCD camera is available, this mode may be the optimal one in many applications, yet to date it is still not widely known or used.

The principal difficulties in CCD flatfielding come from any spectral mismatch between the data and the flatfield. This is the result of two facts: (1) QE response curves differ somewhat from one pixel to another, e.g., due to impurities or thickness variations; and (2) any finite-bandpass image is really a sum of 
monochromatic images, each with its own interference fringe pattern, weighted by the spectrum of the incident light and the bandpass response. This is easily demonstrated by comparing the flatfields obtained with two different narrow-band filters. Consequently, broader bands are harder to flatfield, and the wavelength dependence of the flatfield response also varies spatially on the CCD. This suggests that the future of CCD-based photometry may be in intermediate or narrow band systems in which the placement of the bandpasses is motivated by some astrophysical reason (e.g., Gunn-Thuan, Stromgren, etc.), rather than the sensitivity of obsolete photocathodes. Moreover, in pixels where the object signal is comparable to, or greater than the sky foreground (i.e., for almost anything brighter than $\sim 21^{m}$, and in particular for the standard stars), this color-dependent variation will vary from one object to another, and can the coefficients of instrumental color equations. This can be a real problem in stellar photometry where the PSF is undersampled, e.g., with HST, and some iterative scheme using different color flatfields may be necessary.

The most common type of flatfields are out-of-focus images of the telescope dome, illuminated by an incandescent lamp. These provide a high signal, and thus low pixel-to-pixel Poisson noise, but this may be highly deceptive; generally, the use of dome flats leads to the worst systematic flatfielding problems due to the frequency response variations discussed above. Some of the difficulties may be caused by red leaks in one's filters (Stetson 1988), but some are simply due to the mismatch between the flatfield illumination spectrum and that of the sky and astronomical objects. Using high-temperature light bulbs and/or blue filters for the dome illumination can help. Dome flatfields often have a residual low spatial frequency variation or a gradient due to an uneven illumination, which can be removed with the use of some kind of a sky flatfield. These can be dawn or twilight sky exposures, which have a high signal and the correct illumination, but colors which may be just as wrong as those of dome flats. Better than this are median-filtered "blank sky" exposures, or sky median stacks. In general, some combination of the dome and sky flats is used in most CCD imaging work. Similar techniques apply for both stare and short scan modes. Typical resulting accuracies are on the order of 0.2 to $2 \%$ above expected Poissonian errors, on all spatial scales, and often the flatfielding is worse in the bluer bands.

The sky median stacks are obtained using a large number (at least 5) of "blank" sky (i.e., free of any bright or extended sources) images, scaled to the same sky mean, with all discernible objects removed or flagged. If there are $N$ such images, a data cube is formed, and at each row and column $(i, j)$, a set of corresponding (non-flagged) pixels from all $N$ images is extracted: $I_{1}(i, j), \ldots$ $I_{N}(i, j)$. The median, or the average of a few values around the median of that set is then an estimate of the unpolluted sky signal at the given row and column $(i, j)$. The quality of the median stack frame, and its pixel-to-pixel Poissonian noise are determined by the number and exposure times of the sky fields used to construct it. Its accuracy is limited by the unremoved faint stars and galaxies, and PSF wings, typically at a $\sim 1 \%$ level, over the scales of tens to hundreds of pixels. A good sky median stack is the "ideal" flatfield for the situations in which the sky signal dominates (i.e., any faint objects work). In practice, it is hard to accumulate enough signal in a given night to have a median sky stack usable as a flatfield on its own, but median stacks for the run are possible. One subtlety worth noting is that one should avoid, if it is at all possible, dividing an object frame by a median sky flat which includes that object frame as part of its constituent 
stack. This will result in some fraction of pixels in the object frame being in effect divided by themselves during the flatfielding process; a distinctive and undesirable spike results in the histogram of resulting pixel values. This can have unpleasant consequences for photometry, and the sky determination.

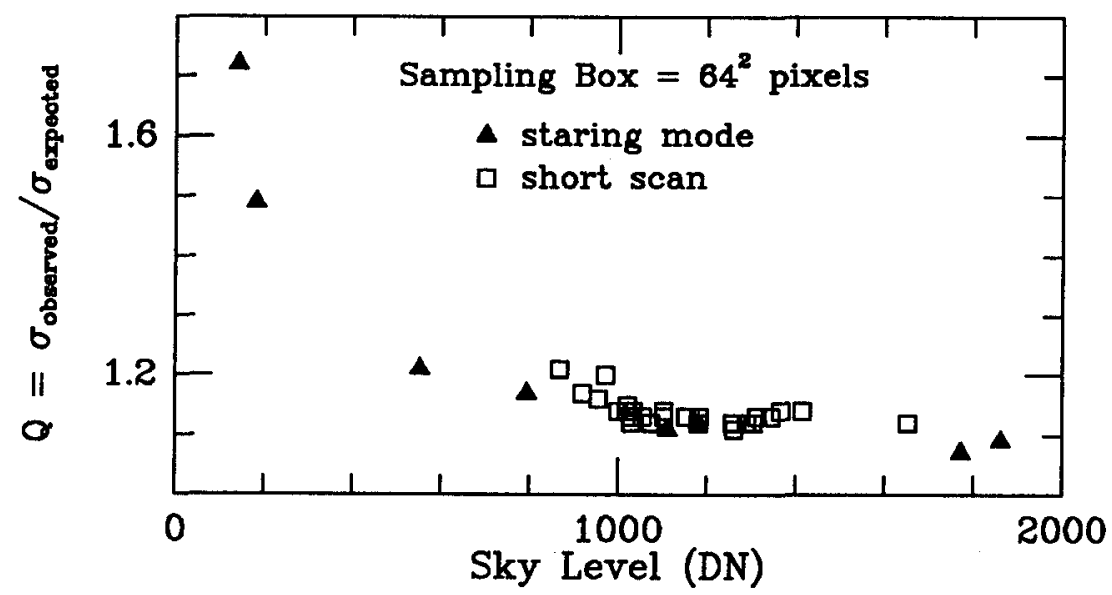

Figure 1. Dependence of the "excess noise" parameter $Q$ on the mean signal level. The data are from a TI $800 \times 800 \mathrm{CCD}$ at the prime focus of the KPNO 4-m telescope, obtained in the $R$ band, and flatfielded with the simple dome flats. The expected noise (Poisson + readout) is $\sim 2.2 \%$ at $500 \mathrm{DN}$, and $\sim 1.1 \%$ at $1000 \mathrm{DN}$ $\left(1 \mathrm{DN} \simeq 4.15 e^{-}\right)$. The triangles are the data obtained in the stare mode, and the squares are the short scans.

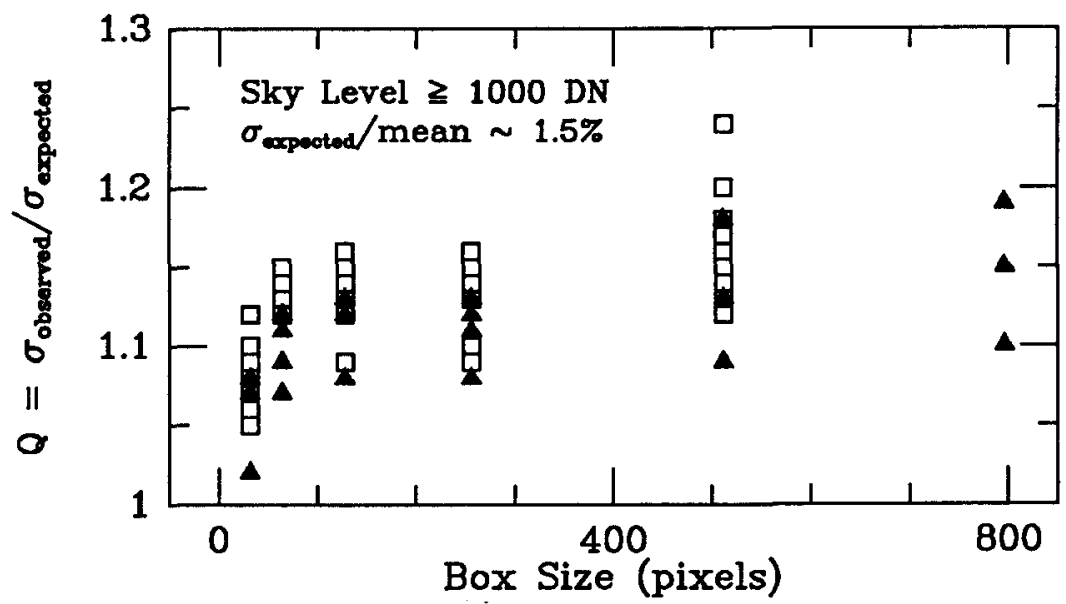

Figure 2. Dependence of the parameter $Q$ on the size of the subimage used to evaluate the data histograms. The data and the symbols are as in Fig. 1, except that the frames are limited to those with mean sky level $\geq 1000 \mathrm{DN}$, and the expected noise is $\sim 1.5 \%$ for all data points. Note that the residual noise is still $\sim 0.2 \%$ above the Poissonian at most spatial scales. 
A good tool for the investigations of flatfielding efficiency are the data histograms. In uncrowded fields, they can be well modeled as Gaussians with dispersion $\sigma^{2}$. Let us define the "excess noise" parameter, $Q=\sigma_{\text {observed }} / \sigma_{\text {expected }}$, where the $\sigma_{\text {expected }}$ accounts for the Poissonian and readout noise. Figure 1 shows dependence of the parameter $Q$ on the mean signal level, for CCD subimages of size $64^{2}$ pixels. The rise at the low light level is probably due to the charge transfer inefficiency and additive errors. Leveling-off at the higher signal levels suggests color mismatching or nonlinearities. Figure 2 shows the dependence of $Q$ on the spatial scale: for a pure Poissonian noise, one would expect $Q$ to go down as more pixels are sampled; instead, it is nearly constant. This suggests that the residual flatfielding noise is of the $1 / f$ type, and a simple reasoning of the type $(S / N) \sim \sqrt{t_{\text {exposure }}}$ is likely to be incorrect.

In the case of drift scans, the column-to-column response can be derived from the data itself, e.g., as column medians, iteratively corrected for any sky brightness variations (row-to-row). This is not feasible if there are large, extended objects in the field, e.g., bright galaxies. An alternative is to generate a column vector from another sky exposure, or from a dome flat, but the color of the latter can be wrong. For faint object imaging in good photometric conditions, the data can be corrected to almost the Poissonian limit accuracy, better than $0.5 \%$, and perhaps as good as $0.1 \%$ above the Poissonian noise.

Flatfielding of spectroscopic CCD data is in principle simpler than that for direct images, because all the pixels are illuminated by a monochromatic signal, and as long as there are no shifts between the pixels and the Angstroms, even the interference fringes stay anchored. An additional problem is presented by the uneven width of the entrance apertures or the slit, but it can be easily corrected using a co-called slit function, derived from sky exposures; cf. Djorgovski and Spinrad (1983) for more details.

Most spectrum-related problems in CCD flatfielding are multiplicative errors, which can in principle be corrected by division by an appropriate correction image. The more insidious limits on flatfielding accuracy are caused by the additive errors, which are much harder to correct. Obviously, presence of additive signals in the raw charge image and/or the flatfield will perturb their division and give an incorrect astronomical object image. There are several possible sources of such additive errors: bias and dark current variations; charge transfer problems, unremoved interference fringes (from the night sky or emission-line objects), scattered light from bright sources, electronic drifts, and possibly polarization dependences of the CCD response. Some of these are fixed properties of a given CCD device, which can be mapped and then removed before flatfielding, e.g., deferred charge columns (Baum, Thomsen, and Kreidl 1981). Others, e.g., bias and dark variations, or electronics artifacts, are preventable through a good hardware design and maintenance, and can be easily monitored. But some problems, e.g., the charge transfer inefficiency, may be scene-dependent and difficult to remove.

The best way of coping with additive errors may be reference sky frame subtraction. Baum, Thomsen, and Morgan (1986) achieved flatfielding accuracies of the order of $0.2 \%$ with this technique. However, the fundamental limit comes is set by the faint stars and galaxies which are by necessity present in the reference sky frames. The procedure is familiar to IR observers, and is essential to the use of all present and near-future IR imaging devices.

Finally, CCD's are highly, but not perfectly linear-response detectors. The measured non-linearities of most types of astronomical CCD's (TI's are better than 
RCA's) are less than $1 \%$, perhaps about $0.01 \%$ for most part of their dynamic range (well below saturation level). Still, ideally the mean flatfield signal level should be close to the mean level of the signal of interest. More insidious is quantum efficiency hysteresis (Griffiths 1985). This is minimized with the new flashgate technology: platinum coating, oxygen soaking, UV flooding, and other magick; some elixirs and physics are described by Janesick et al. $(1986,1987)$.

Other sources of flatfielding errors may include moving dust specks, and their out-of-focus shadows. The problem can be rather bad in the cameras with many optical surfaces and a considerable flexure. If computer time is no objection, and there are flatfields taken at different times and telescope pointings, one can try to separate the time-dependent and time-independent factors in a flatfield. A much better alternative is to have a well-designed and clean CCD camera.

How well can one do? The commonly used methods (e.g., sky-corrected dome flats) generally achieve flatfielding accuracies on the order of $0.5-2 \%$ in for a broad-band image taken with either steady or short scan modes. Sky medians are hard to make better than about $1-2 \%$, because of the residual faint objects or PSF wings. In narrow bands, with dome or dawn/twilight sky flatfields, and with reference sky subtraction, one can reach $\sim 0.1 \%$. Self-flatfielded drift scans may approach the Poissonian limits, but that has been tested only at the 0.1 $0.5 \%$ level so far; besides, drift scans are not practical for most standard star observations. Thus, it appears that the present limits are reaching a plateau of flatfielding accuracy at $\mathrm{a} \sim 0.1 \%$ level above the expected Poissonian noise, on all spatial scales. For averaging over many pixels, one may be able to do slightly better $\left(0.0005^{m}\right.$ ?). However, in order to push the accuracy further, it may be necessary to obtain better, and not merely bigger, CCD's.

\section{REFERENCES:}

Baluteau, J.-P., and D'Odorico, S. (editors) 1986, Proceedings of the ESO-OHP Workshop on The Optimization of the Use of CCD Detectors in Astronomy, ESO Conference Proceedings No. 25.

Baum, W., Thomsen, B., and Kreidl, T.J. 1981, Proc. S.P.I.E. 290, 24.

Baum, W., Thomsen, B., and Morgan, B. 1986, Astrophys. J. 301, 83.

Djorgovski, S., and Spinrad, H. 1983, in Proceedings of the AAS/OSA Joint Topical Meeting on Information Processing in Astronomy and Optics, June 1983, St. Paul, Minn., p. ThB2-1. AAS/OSA publication.

Djorgovski, S. 1984, in Proceedings of the Workshop on Improvements to Photometry, eds. W. Borucki and A. Young, p. 152. NASA CP-2350.

Griffiths, R. 1985, STScI Newsletter, October 1985, p. 5.

Hall, P., and Mackay, C. 1986, M.N.R.A.S. 210, 979.

Janesick, J., Elliot, T., Daud, T., and Campbell, D. 1986, Proc. S.P.I.E. 627, 543. Janesick, J., Campbell, D., Elliot, T., and Daud, T. 1987, Opt. Eng. 26, 852.

Mackay, C. 1986, Ann. Rev. Astron. Astrophys. 22, 255.

Stetson, P. 1988, DAO preprint; and this volume.

Stetson, P., and Harris, W. 1988, Astron. J. 96, 909.

Tyson, J.A. 1988, Astron. J. 96, 1.

Wright, J.F. 1982, Ph.D. Thesis, University of Cambridge. 